\title{
Journey through quantitative haematology
}

\author{
Dr Clarence CK Lam, FHKAM (Pathology), FHKCPath (Haematology)
}

Invited author, Hong Kong Museum of Medical Sciences

https://doi.org/10.12809/hkmj-hkmms202012

We tend to remember pathology, putting aside autopsies and specimens which may not be everybody's cup of tea, as beautiful pictures captured from histological sections under the microscope. Pathology, in fact, has since many years ago evolved beyond images. Something more precise with minimal inter-observer difference is clearly more desirable, and nothing is more precise than numbers. Therefore, quantifying what we can see, grossly or microscopically, remains one of the most important missions of the pathologists.

Let us start with cell counting in haematology. In modern clinical practice, full blood count is the most basic haematology test provided by any decent laboratory round the clock, and the results can be generated by elaborate automated haematology analyser systems literally within minutes according to the clinical conditions of the patients. Hardly can one imagine the enormous efforts and time associated with a report on the 'not-so-full' blood count before the advent of haematology analysers based on the Coulter principle discovered by Wallace $\mathrm{H}$ Coulter in the late 1940 s for which a patent was granted in 1953.

Blood counts were performed with a haemocytometer in the good old days. The haemocytometer, more commonly known in the laboratory as a counting chamber, or simply the chamber, was invented in the late 19th century. Its invention is credited to Louis-Charles Malassez (1842-1909), a French anatomist and histologist. The most commonly used haemocytometer in the haematology laboratory is the Neubauer counting chamber, which is shown in Figure 1. This is a generous gift from Professor James B Gibson to the Hong Kong Museum of Medical Sciences, as something that he himself had used in his earlier career, and which he had carefully kept until his retirement. It is essentially a thick glass microscopic slide with a chamber of specific depth, etched accurately at a defined dimension with a grid of perpendicular lines. A properly positioned special coverslip with certified thickness and flatness, together with the best technique of loading an appropriately diluted and thoroughly mixed blood specimen by pipettes, ensures the precision of the volume. By counting the number of cells in defined areas of the haemocytometer, the number of cells in a specific volume of the diluted blood specimen is known. The concentration of cells in the blood overall, or cell counts, can be calculated by correcting for the dilution.

The graduated glass tubes with a bulge (Fig 2), which harbours a little stirrer of either red or white, are actually pipettes for diluting the peripheral blood specimen and loading of the counting chamber: the one with the red stirrer is for diluting the blood specimen for counting of red cells while the white is for leucocytes and platelets. These pipettes had been routinely filled by suction by mouth through a rubber tube until the risk of mouth pipetting was recognised, and filling by aspirating with small volume syringes was introduced. Different diluents and dilutions, with which you are not going to be bothered, are used for counting of different cell types. After dilution and thorough mixing on a device and discarding the portion of the specimen below the bulge, the diluted blood specimen is loaded through capillary action to the chamber. Any pressure will

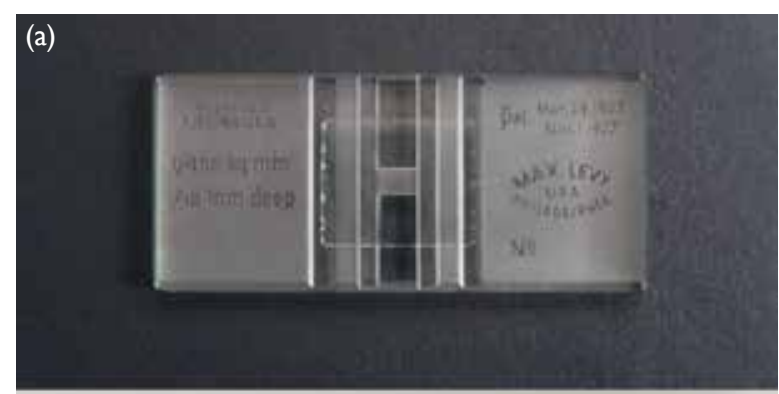

(b)

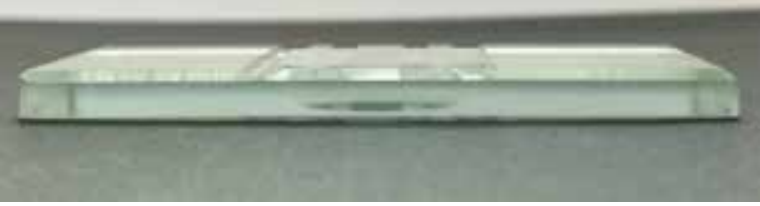

FIG I. Neubauer haemocytometer viewed (a) from the top and (b) from the side

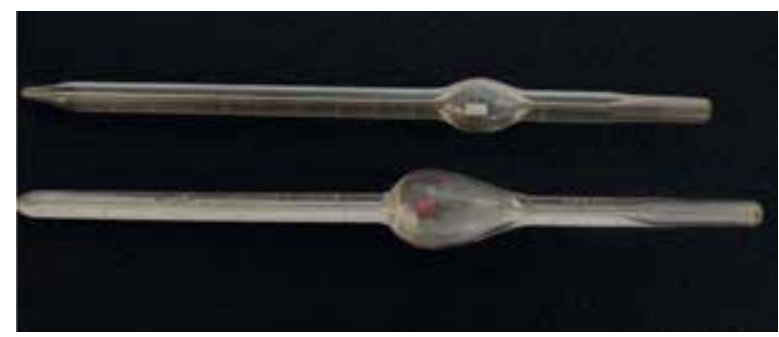

FIG 2. Diluting pipettes for use with the haemocytometer 
introduce too much specimen into the counting chamber and result in an erroneous volume, thus a wrong cell count. Counting under the microscope will be performed after letting the loaded counting chamber settle for a standard period of time, usually 5 to 10 minutes. If the loaded counting chamber is left for too long before counting, the specimen may evaporate and dry out, particularly in laboratories of low humidity, thus affecting the distribution of the cells, and resulting again in an erroneous result.

It is not difficult to imagine how tedious it was to generate just one report on blood counts. It was certainly a much more challenging task organising a laboratory haematology service with such manual tests. You would be surprised to learn that many a time it was the house officers' or the residents' job to do the blood counts. How different life was in the medical profession just a few decades ago! Mind you, we have yet to touch on the quality assurance measures which are of pivotal importance in patient care. The prevailing quality assurance framework then was understandably inadequate when compared with the current concepts and understanding of total laboratory management, particularly concerning the requirements and standards of laboratory accreditation. The first steps to ensure 'correctness' of the results were to do the tests in duplicate and standardise as far as possible the various manual steps.

The last two decades of the 20th century saw rapid evolution of cell counting technology. Incorporating some relatively basic automation, this evolution enabled comparatively high throughput quality service, with remarkable accuracy and precision, to cope with the increasing volume and diversity of clinical service. Improvements in cell counting technology, albeit in a more incremental manner, are still seen in the first two decades of the 21st century. However, efficiency, with enhanced workflow design and post-analytical software support, and flexibility in setup, with large-scale or modular automation to suit laboratory services of variable complexities, have been the main foci of development. These new automated analyser systems also have built-in quality assurance capabilities to cater for the requirements of accreditation.

The counting chamber is very seldom or hardly ever used nowadays for blood counts in the haematology laboratory because modern automated haematology analyser systems have different modes of analysis for accurately quantifying different cell types in the peripheral blood should there be any need. It is still being used in the clinical laboratory setting for cell counting in different kinds of body fluids. Even for this purpose, its use is again on a downward trend, as new analysers can also cater for cell counting of many body-fluid types.

I believe it is appropriate to share with you some information about Professor Gibson, the donor of this artefact. Professor Gibson graduated in 1943 from the University of Edinburgh and also received his MD from Western Reserve University at Cleveland, United States the same year. After serving in the Royal Navy, during which he took part in the Normandy landing, he worked on both sides of the Atlantic before taking up an appointment as Professor and Head of Department of Pathology at The University of Hong Kong (HKU) from 1963 to 1983. He also served as Dean of the Faculty of Medicine from 1972 to 1978 . In addition to being a world recognised expert in liver pathology, Professor Gibson had contributed extensively in shaping and driving for advancement in medical care in Hong Kong through upgrading the standard of pathology practice and service. It is largely due to the phenomenal efforts and dedication of Professor Gibson that pathology practice as we know today took its shape. Professor Gibson established a separate Department of Microbiology in 1968, set up an immunology section and cytology service in the 1970s, set up a tissue typing service, and oversaw the evolution of the Clinical Biochemistry Unit into a separate unit in the early 1980s. He also organised improvements in various facilities. He is particularly remembered for setting up a central electron microscope unit in HKU and opening of a new Clinical Pathology Building in Queen Mary Hospital in 1972. He was instrumental in the formation of the HKU Hospital Pathology Service in Queen Mary Hospital which had provided a high-quality pathology service until it was taken over by the Hospital Authority in the 1990s. Professor Gibson's trainees were the first in Hong Kong to pass the Royal College of Pathologists Membership examination. Not only did pathologists benefit from the foresight of Professor Gibson, training of technical professionals throughout Hong Kong was also upgraded through his oversight of the establishment of a territory-wide medical laboratory technician training programme, the Ordinary and Higher Certificates in Medical Laboratory Technology, via the Extramural Department (now SPACE) of HKU. Professor Gibson received an Honorary Doctorate of Science from HKU on his retirement in 1983, and continued to keep an interest in the pathology profession in Hong Kong after his retirement and relocation to Scotland.

The haemocytometer has certainly done its due in fulfilling its mission in the history of laboratory haematology practice. It is an integral component of the science and art of cell counting. It is a delight to know that cell counting, although in areas outside clinical laboratory practice, using a haemocytometer can be automated!

\section{Acknowledgement}

I would like to thank Professor Faith CS Ho and Professor SC Tso for their invaluable input. 